\title{
Puntos de vista científicos en las series de televisión*
}

\author{
Scientific points of view in TV series
}

LAURA GARCÍA DÍAZ**

\begin{abstract}
Resumen: Kitcher propone una democratización de la ciencia que insiste en la necesidad de que el público se involucre en la práctica científica. Esto permite analizar el valor de algunas series televisivas como medio divulgador que permite generar opiniones sobre los desarrollos científicos actuales y sus repercusiones en el futuro. A través de la definición que nos da Moline sobre los puntos de vista analizaremos la forma en que las series proyectan diferentes perspectivas que permiten al público acercarse a problemas científicos o, en términos de Kitcher, formar una opinión responsable sobre el conocimiento científico y sus posibles aplicaciones tecnológicas.

Palabras clave: Puntos de vista, Democratización, Ciencia, Series TV, Tecnología.
\end{abstract}

\begin{abstract}
Kitcher proposes a democratization of science that insists on the need for the public to be involved in scientific practice. This allows us to analyze the value of some television series as a disseminating medium that allows to generate opinions about current scientific developments and their repercussions in the future. Through Moline's definition of points of view, we will analyze the way in which the series project different perspectives that allow the public to approach scientific problems or, in Kitcher's terms, to form a responsible opinion about scientific knowledge and its possible technological applications.
\end{abstract}

Key words: Points of view, Democratization, Science, TV Series, Technology.

\section{Democratización de la ciencia}

La filosofía de la ciencia desarrollada en el siglo XX se caracteriza, sobre todo, por haber introducido en sus análisis un elemento hasta el momento ignorado: lo histórico. Esto abrió un panorama completamente nuevo y dio lugar en los años 70 y 80 a los estudios sobre la ciencia desde un punto de vista sociológico, antropológico, económico, de género o de políticas científicas. A diferencia de lo que había estado haciendo la filosofía tradicional de

Recibido: 30/06/2018. Aceptado: 21/10/2018.

* Artículo realizado en el marco del proyecto Puntos de vista, disposiciones y tiempo. Perspectivas en un mundo de disposiciones (FFI2014-57409-R), financiado por el Ministerio de Economía y Competitividad del Gobierno de España).

** Universidad de La Laguna. Investigadora predoctoral. E-mail: lauragd10114c@gmai.com. Líneas de investigación: Filosofía de la ciencia, Estudios sociales de la ciencia, Filosofía y cultura popular. Tiene en prensa: Vazquez, M. y L. García (2018), "Perspectivas temporales", en: Vázquez, M. y M. Liz, Tiempo y perspectiva. Barcelona: Laertes. García, L. y A. Hernández (2018), "El papel de las series de televisión en la democratización de la ciencia", en: Proceedings IX Conference of the Spanish Society for Logic, Methodology and Philosophy of Science, Madrid: UNED. 
la ciencia, centrada mayoritariamente en aspectos metodológicos o normativos, estas nuevas tendencias vuelcan sus análisis sobre todo en la práctica científica. Esta dimensión práctica de la ciencia, y todo aquello con lo que se relaciona, ha sido pensada, entre otros y otras, por Philip Kitcher, el cual defiende la necesidad de comprender el papel de la ciencia en una sociedad democrática. Kitcher se suma así al debate en torno a la "democratización de la ciencia" abierto por Paul Feyerabend en los años 70. Uno de los elementos centrales dentro de dicho debate es el papel del sujeto.

Kitcher (2010) resalta la necesidad de que el público, en un sentido amplio, se involucre en la práctica científica. Siguiendo a Robert Dahl, señala la imposibilidad de proporcionar a la ciudadanía de las democracias contemporáneas una medida de control sobre las decisiones que les afectan y que respondan a su esquema de valores. La democracia, que surge como respuesta al problema de la opresión, se encuentra ahora ante la imposibilidad de representar por completo los intereses de su ciudadanía, más aún teniendo en cuenta la persistencia de opresiones difícilmente identificables. Gran parte de las decisiones que afectan a la población son demasiado complejas para que los individuos formen una opinión responsable al respecto. Además, los representantes políticos no tienen posibilidad, según Kitcher, de saber si las políticas que incentivan responderán a las necesidades de la población, una situación que se complica aún más en la comunidad científica. De esta manera, Kitcher nos propone pensar modos en los que instituciones alternativas puedan permitirnos llegar a su ideal de "ciencia bien organizada", un modelo que depende de deliberaciones basadas en preferencias que responden tanto a la comprensión de las oportunidades disponibles como a las necesidades expresadas. Para Kitcher es un error dar por hecho el valor intrínseco de la ciencia para comprender nuestro mundo si no se pone antes de manifiesto que aquello que denominamos "conocimiento puro" sólo está disponible para un número reducido de personas y el poco interés por hacer accesible a todos "nuestra herencia científica".

Es por esto que en este artículo se propone resaltar el papel democratizador de ciertos medios de producción cultural que permiten al público acercarse a algunos problemas científicos o, en términos de Kitcher, permiten formar una opinión responsable sobre conocimiento científico y sus posibles aplicaciones tecnológicas. La propuesta de ciencia bien organizada exige, entre otras cosas, que la defensa del conocimiento puro provenga de deliberadores que comprendan las diferentes opciones y que incorporen las necesidades de los otros. Este aspecto permite analizar el valor de algunas series de televisión como un medio para instruir al público. La democratización de la ciencia que pide Kitcher puede estar dándose como subproducto a través del fenómeno actual de las series de televisión. Éstas han dado lugar a un nuevo tipo de cultura audiovisual, así como a un perfil de espectador interesado por tramas de índole científica y distópica que reflexiona sobre temas que, hasta entonces, podían resultarle desconocidos. A través de la noción de punto de vista, o perspectiva, veremos cómo una serie de televisión permite acceder a cuestiones que amplían la imagen que tenemos de la realidad.

\section{Series y divulgación científica}

A partir del siglo XIX la ficción literaria comienza a narrar historias fantásticas y hechos insólitos apoyándose en los descubrimientos científicos de la época. La ciencia 
ficción se define como un género en el que se relatan historias de corte fantástico a través de una explicación racional. El avance de la ciencia y la técnica hizo que, de alguna forma, los escritores crearan narraciones en las que el poder del ser humano y su control sobre la naturaleza permitían traspasar los límites de lo razonable. La novela Frankenstein (1823) de Mary Shelley es una de las primeras obras que dan origen a lo que se conoce como literatura de ciencia ficción. En ella se narra cómo un científico, Víctor Frankenstein, crea un ser monstruoso a partir de restos de cadáveres. Otras novelas como El extraño caso del doctor Jekyll y Mr. Hyde (1886), de Robert Louis Stevenson, Veinte mil leguas de viaje submarino (1869) de Julio Verne o La guerra de los mundos (1898) de Herbert George Wells serán también incluidas en este género. En ellas vemos historias sustentadas en las inquietudes científicas de la época y un intento de predecir cómo la ciencia avanzaría en el futuro. En definitiva, la ciencia ficción se ha constituido como un género literario que permite especular sobre el futuro de la ciencia y la humanidad a partir del conocimiento del presente. Siguiendo a Miquel Barceló (2005) podemos entender este género de la siguiente manera: "la ciencia ficción es una narrativa que nos presenta especulaciones arriesgadas $\mathrm{y}$, muy a menudo, francamente intencionadas que nos hacen meditar sobre nuestro mundo y nuestra organización social o sobre los efectos y las consecuencias de la ciencia y la tecnología en las sociedades que las utilizan" (Barceló, 2005, 3).

En las décadas de los 40 y los 50 del siglo XX, este género literario adquiere especial relevancia. Escritores como Isaac Asimov, Arthur C. Clarke o Robert A. Heinlein, lo llevan a otro nivel debido a la rigurosidad y exhaustividad que les aporta su formación científica. Ellos y otros autores de ciencia ficción muestran en sus obras "las capacidades del científico, del divulgador y del novelista de ciencia ficción" (Barceló, 2005, 3). Así, las historias de ciencia ficción que surgen a partir del siglo XIX y que adquieren una gran relevancia en el siglo XX comienzan a trasladarse a otros géneros como el cómic, el cine y la televisión. A principios del siglo XX aparecen los primeros ejemplos de cómics o historietas de ciencia ficción, como Buck Rogers (1929), Brick Bradford (1933) o Flash Gordon (1934). En el cine esto ocurre incluso un poco antes. A pesar de los escasos medios técnicos de la época, en 1902 Georges Méliès estrenó Viaje a la Luna, una película de apenas 14 minutos basada en dos novelas de ciencia ficción: De la Tierra a la Luna (1865), de Julio Verne, y Los primeros hombres en la Tierra (1901), de Herbert George Wells. Con el avance de la tecnología y la espectacularidad que van ganando los efectos especiales, el cine se ha convertido en un vehículo especialmente adecuado para la especulación sobre el uso de la ciencia y la tecnología. También la televisión incorpora en su catálogo narraciones de este tipo, que se han visto, como el cine, favorecidas por el desarrollo de los medios técnicos. En 1966 se estrena en EEUU la serie Star Trek ${ }^{l}$, una de las pioneras de la ciencia ficción en la pequeña pantalla. En esta serie podemos ver una recreación de múltiples dispositivos tecnológicos que años más tarde se convirtieron en realidad, como las pantallas táctiles, las PDA, los teléfonos móviles o, incluso, la comunicación inalámbrica entre ordenadores, que fue creada apenas cuatro años más tarde del estreno de la serie. Otras series de éxito fueron Perdidos en el espación, Buck Royers en el Siglo

1 NBC: 1966-1969.

2 CBS: 1965-1968. 
$X X V^{3}$ o Battlestar Galactica ${ }^{4}$. Como vemos, las series de televisión, al igual que el cine y la literatura, permiten dar a conocer puntos de vista científicos. Sin embargo, se debe recordar que la ciencia ficción, como el resto de la ficción, no es un relato estrictamente realista que tenga como hilo conductor una teoría científica, sino que consiste en presentar un relato en el que se especula sobre la ciencia, la tecnología, su desarrollo y sus usos. A la vez que encontramos series que permiten divulgar conocimiento e incentivar la reflexión a través de su trama, otras puede que se acerquen más al género literario fantástico y que divulguen más bien la pseudociencia. Pero no es este el tema que nos ocupa. El objetivo de este trabajo se centra en mostrar el valor de aquellas en las que se permite divulgar puntos de vista científicos o, al menos, incentivar la reflexión sobre estos. El que algunas series contribuyan más a la divulgación de la pseudociencia que de la ciencia es algo que no debe lastrar el valor pedagógico de estas producciones culturales.

Como decíamos, la ciencia ficción, en tanto que narración que reflexiona sobre el desarrollo de la ciencia y la tecnología, sigue estando presente en múltiples productos culturales, y su valor pedagógico ha sido ampliamente reconocido. En 1970 se crea la Science Fiction Research Association, en la que profesores de todo el mundo atienden a las posibilidades didácticas de este género. Con el mismo objetivo, la Universidad del Estado de Kent en Ohio crea en 1959 una revista, Extrapolation ${ }^{5}$, a ésta se suman otras revistas académicas como Foundation ${ }^{6}$ o Science Fiction Studies ${ }^{7}$. En las últimas décadas, las series de televisión han incorporado cada vez más en sus tramas esta capacidad divulgadora que encontramos en la ciencia ficción. Uno de los motivos puede ser precisamente que éstas ya no se asocian ni surgen únicamente al dispositivo transmisor del que surgieron, la televisión. Las series son ahora producidas y emitidas por plataformas de internet como Netflix, HBO o Amazon Prime que encuentran inversión en los propios consumidores, lo cual ha permitido que la producción de series alcance mayor excelencia con respecto al contenido y calidad artística. En la actualidad encontramos muchas series que introducen puntos de vista científicos, como Fringe ${ }^{8}$, Black Mirror $^{9}$ o Westworld ${ }^{10}$, aunque no todas ellas pertenezcan al ámbito de la ciencia ficción, como The Bing Bang Theory ${ }^{11}$ o The Knick ${ }^{12}$. En este tipo de series encontramos algo novedoso, puesto que consiguen incentivar la reflexión sobre la ciencia y la tecnología de una manera un tanto diferente a como ha ocurrido en la literatura y el cine.

$\mathrm{Al}$ igual que el cine, las series son capaces de presentar temas complejos e incentivar la reflexión de una forma lúdica y aprovechando la espectacularidad de los medios técnicos. Sin embargo, lo que quizás hace a las series un medio idóneo para conseguir una democratización del conocimiento y lo que las diferencia de otras formas de divulgación es su

3 NBC: 1979-1981.

4 ABC: 1978-1980.

5 Kent State University Press.

6 Macmillan Publishers.

7 DePauw University.

8 Fox: 2008-2013.

9 Chanel 4: 2011-actualidad.

10 HBO: 2016-actualidad.

11 CBS: 2007-actualidad.

12 Cinemax: 2014-2015. 
capacidad para enganchar al espectador, despertar su empatía y hacerle adoptar el punto de vista proyectado en su trama de una forma más intensa y durante un periodo de tiempo más largo. Al igual que las novelas por entregas de Dickens, Trollope o George Elliot, las series de televisión generan suspense a lo largo de la obra. Brett Martin (2014) nos dice que, aunque siempre se haya relacionado las series de televisión con el cine, podemos encontrar en ellas algo similar a las novelas por entregas: "la televisión se ha comparado de manera reflexiva con el cine, pero esta forma de narración continuada y sin final estaba, por utilizar una comparación habitual, más próxima a las novelas victorianas por entregas, otra explosión de alta cultura en un medio popular vulgar" (Martin, 2014, 20).

Concepción Cascajosa (Cascajosa, 2016) ha señalado que desde hace algunos años ha surgido un nuevo panorama de fragmentación de audiencia con respecto a las series en el que aparece un lugar específico para un perfil sofisticado de espectador, al que le interesa la innovación genérica, las temáticas actuales y la representación sin prejuicios de ciertos tabúes. Podría decirse que este perfil de espectador puede asociarse, además, a otros ámbitos, como a las nuevas formas de periodismo digital promovidas por plataformas como YouTube y blogs que permiten que cualquiera con acceso a internet pueda no solo comunicarse sino depositar en ellas información o, incluso, productos culturales. Este espectador recibe, a menudo con gran entusiasmo, contenido científico y filosófico que por lo general parece más asequible y atractivo que el contenido ofrecido por las oportunidades de aprendizaje presentadas en el ámbito académico.

Es por ello que puede pensarse que el déficit de democratización de la ciencia señalado por Kitcher (2010) puede ser de cierta forma nivelado a través de las series, éstas son capaces de crear narraciones y transmitir el contenido que debería ser divulgado por la propia comunidad científica o política. Se trata de un medio capaz de proyectar el resultado de ciertas investigaciones científicas, es decir, más allá de llevar al espectador un conocimiento que le resulta desconocido, es capaz de incentivar el cuestionamiento de las prácticas y fines en la ciencia y de especular sobre su desarrollo. En un contexto en el que la ciencia se ha privatizado, las series pueden servirnos como un recurso para tomar conciencia, esto es, ejemplificar, problematizar y generar opiniones sobre los desarrollos científicos actuales y sus repercusiones en el futuro, tal y como entiende Kitcher que sería necesario en una sociedad democrática. Por ejemplo, algunas facetas de la inteligencia artificial que están aún desarrollándose, ya existen en el plano del imaginario audiovisual y contamos con múltiples proyecciones y problematizaciones al respecto. En cierta forma, las series de televisión han ocupado el privilegiado puesto que hasta ahora había pertenecido al cine y, además, han adquirido un formato y contenido que las convierten en algo así como literatura televisada.

Muchos son los autores que han hablado de los medios de masas y la repercusión e impacto que generan en los espectadores. Ya Adorno (1947) mostraba preocupación por la uniformización que encontraba en el cine y, en general, en una cultura de masas que comenzaba a hacerse latente en torno a la década de los 40, mientras que, a la vez, hacía hincapié en la potencialidad emancipadora que albergaba.

Quizás hoy podríamos decir que las series han ido adquiriendo la necesidad que señalaba Adorno (Adorno, 1947,15) de unir la utilización de la técnica con la capacidad de expresar la realidad; de hacer llegar ciertas ideas a los espectadores, de desarrollar tramas complejas 
y lo que es aún más interesante: crear personajes y puntos de vista profundos que podemos llegar a conocer y comprender. También lo que dice Rancière (2008) sobre las artes escénicas puede ser trasladado a las series para entenderlas como un producto artístico emancipador. A su parecer, el teatro, al igual que el resto de artes escénicas que requieren de un espectador, ha sido redefinido, el espectador deja ser pasivo si se entiende que su poder es retomado y reactivado en la performance, en la inteligencia y en la energía que se reproduce en ella. Esto supone pensar que el embrutecimiento del espectador se solventa al mostrarle en la obra lo extraño, lo inusual, en definitiva, un enigma al que debe encontrar sentido (Rancière, 2008, 11-12).

Dominique Wolton nos dice, con relación a los medios de comunicación individualizados e interactivos, que la televisión no ha estado nunca tan desvalorizada como hoy en día. No obstante, entiende que las innovaciones técnicas pueden hacernos pasar de la sociedad industrial a la sociedad de la información, en la que lo esencial sea la comunicación. La televisión se constituye, según este autor, como un medio de comunicación hacia la cultura y la democracia de masas, algo que también puede ser dicho de internet y el intercambio de información que propicia.

Pensamos en un emisor movido por las más negras intenciones, y en un destinatario siempre dispuesto a creer en lo que se le cuenta, sin autonomía ni distancia crítica. Negamos tanto la distancia crítica del receptor como la dimensión normativa del emisor, es decir, la posibilidad de una cierta intercomprensión. (Wolton, 1999, 42)

Otros autores como Pierre Bourdieu se muestran escépticos con respecto a esta idea. Bourdieu (1996) afirma que, por lo general, la televisión se ha establecido como un medio en el que se somete el mensaje a la forma, es decir, un medio en el que los investigadores, científicos o escritores deben sacrificar el discurso reflexivo para conseguir que sea, principalmente, atractivo. Bourdieu recupera el "Ser es ser visto" de Berkley para explicar cómo la televisión permite dar a conocer a las figuras académicas, a pesar de que el mensaje, lo que dicen, pase desapercibido. Aunque desconfía en la forma actual en la que se hace televisión, nos dice que en determinados casos aparecer en ella puede constituir una especie de deber "la televisión es un instrumento que, teóricamente, ofrece la posibilidad de llegar a todo el mundo" (Bourdieau, 1996, 18) y es que entiende que entre las más importantes misiones de los científicos está la de hacer llegar a todos los logros de la ciencia. Aunque actualmente la producción de series comience a desligarse de la televisión, ésta sigue siendo un medio de comunicación masivo, y a pesar de las críticas que podemos encontrar sobre televisión y el cuestionamiento del tipo de mensaje que se reproduce en ella, lo que podemos quizás afirmar es que en las series encontramos una anomalía. En éstas se produce una unión del formato atractivo, visualmente seductor, con una narración que recupera lo mejor de la literatura y que, a su vez, permite visibilizar o divulgar contenido académico.

Obviamente, no podemos decir esto de todas las series, pero sería un error no atender al éxito cada vez mayor de series que tocan temas controvertidos y que sobrepasan las expectativas del contenido que esperamos encontrar en ellas. Las series aparecen como un aparato divulgador del conocimiento al mostrar y cuestionar algunos de los desarrollos científicos y 
tecnológicos más controvertidos. El mundo de la imagen, nos dice Bourdieu, está dominado por palabras que hacen ver lo que se nos presenta. Estas palabras pueden crear temor, fobias o, por otro lado, conseguir representaciones sobre un determinado tema; ilustrarnos a través de representaciones sobre las diferentes perspectivas con las que se enfoca una problemática y que nos permiten ampliar nuestra realidad.

En este punto, podemos, por tanto, pasar a analizar cómo una serie de televisión nos permite tener en cuenta los diferentes puntos de vista en relación a una controversia o desarrollo científico, o incluso, mostrar diferentes perspectivas y formas de entender la moral o la política, lo cual es importante para conseguir representar más dimensiones de las que la apariencia ordinaria nos ofrece.

\section{Jon Moline y la noción filosófica de punto de vista}

Analizar la posibilidad de encontrar en las series una forma de democratización o, al menos, de divulgación del conocimiento, puede ser una forma de entender cómo se relaciona la ciencia y la filosofía. Manuel Liz (Liz, 2012) afirma que la filosofía es reflexión sobre el mundo y nuestra relación con él, pero también reflexión sobre la ciencia y sobre lo que nos hace humanos. Series actuales como Black Mirror, Master of sex ${ }^{13}$ o The Knick versan sobre esto. Ofrecen al espectador la oportunidad de reflexionar sobre temas que, por lo general, no le resultan cercanos como, por ejemplo, qué es aquello que nos diferencia de una máquina, qué nos constituye como humanos o cómo podemos entender el desarrollo de ciertas tecnologías. Mediante este producto cultural vemos cómo se representan controversias, repercusiones positivas y negativas, la relación entre ciencia y política o incluso el propio desarrollo histórico de la ciencia. Se trata de un medio audiovisual capaz de proyectar diferentes puntos de vista que pueden resultar relevantes para que los individuos se formen una opinión responsable.

Para mostrar por qué una serie de televisión consigue mostrar desde diferentes perspectivas la realidad o, concretamente, una controversia científica, atenderemos primero a qué podemos entender por puntos de vista. Podemos decir que Ortega y Gasset es, posiblemente, el primer autor considerado perspectivista dado que habló de una posición filosófica que podía ser denominada perspectivismo. Su posición al respecto se ve especialmente en $E l$ tema de nuestro tiempo (Ortega y Gasset, 1923), concretamente en el apartado "La doctrina del punto de vista". Para este autor, el mundo no es material ni espiritual, sino que es un conjunto de perspectivas y la única forma de comprender o acceder a la realidad será desde circunstancias concretas: "todo conocimiento lo es desde un punto de vista determinado" (Ortega y Gasset, 1923, 105), lo cual, supone que uno adopta un punto de vista que, a su vez, excluye otros: "de la infinidad de los elementos que integran la realidad, el individuo, aparato receptor, deja pasar un cierto número de ellos, cuya forma y contenido coinciden con las mayas de su retícula sensible" (Ortega y Gasset, 1923, 103). Ortega señala así cómo desde un determinado punto de vista algo es relevante mientras que, para otro, lo mismo puede pasar totalmente desapercibido.

13 Showtime: 2013-2016. 
Para acercarnos a una definición del punto de vista recurriremos a continuación al pensamiento de Jon Moline ${ }^{14}$. Este autor nos habla de la dificultad de establecer una respuesta clara a la pregunta sobre qué constituye un punto de vista y cuestiona la definición de Kurt Baier, quien entiende que podemos definirlo como la adopción de ciertos principios que nos permitan responder a preguntas prácticas. La discusión entre Moline y Baier se produce en los años 60, y sin embargo no ha dejado de ser recurrente a la hora de hablar de puntos de vista, tanto, que es recogida en obras recientes que trabajan sobre este tema como, como en Liz (2013), Colomina-Almiñana (2018) y Vázquez y Liz (2015). Moline nos dice que la forma en que entiende Baier los puntos de vista a partir de principios, al estilo kantiano, es muy estrecha y acarrea dificultades. Adoptar un punto de vista, a su parecer, supone mucho más que seguir una serie de principios; implica contar con una serie de vivencias. Entiende que la definición de Baier de un punto de vista como un conjunto de principios podría ser conveniente para quien adopte un punto de vista moral pero no para todas las preguntas prácticas y, recurriendo a John Howard Griffin en Black like me, nos muestra cómo intentar adoptar el punto de vista con el que vive, por ejemplo, una persona negra está más relacionado con ciertas experiencias o actitudes que con principios. La expresión adoptar o tomar un punto de vista adquiere, a su parecer, dos sentidos diferenciados que pueden ser útiles para comprender en qué consisten o cómo definirlos.

Por un lado, podemos comprender un punto de vista como una referencia o ubicación espacial, tal y como podríamos entender el punto de vista que toma una cámara al capturar su objeto. Así, podemos entender un punto de vista como un determinado punto de ubicación o emplazamiento desde el cual uno puede ver ciertas cosas o realizar determinadas tareas, pero no otras. Algunos puntos de vista pueden ser adoptados por una sola persona a la vez, como por ejemplo ocurriría desde una montaña cuyo pico sea muy estrecho. Otros, sin embargo, pueden ser suficientemente anchos como para ser compartidos y que diferentes personas puedan adoptar el mismo enfoque o perspectiva a la vez. Que un punto de vista pueda o no ser compartido por más de una persona depende, en este caso, de factores como la elevación, las obstrucciones visuales, la distancia, el contorno del terreno, etc.

Por otro lado, Moline nos habla de otro sentido en el que considerar un punto de vista que adquiere relevancia filosófica. Al igual que los puntos de vista que dependen de la ubicación, los puntos de vista en un sentido filosófico pueden ser adoptados por una sola persona o por una colectividad. Por ejemplo, el punto de vista del presidente es único, mientras que el punto de vista que adopta un militar puede ser ampliamente compartido. Sin embargo, a diferencia de lo que ocurría con la ubicación, Moline piensa que el punto de vista de un militar o del presidente no se conforma únicamente mediante la ubicación que estos ocupan. Lo que le parecerá fundamental sobre un punto de vista no es ya el lugar sino la forma en la que algo es enfocado. Para explicar esto recurre de nuevo a un ejemplo y nos hace pensar en cómo un policía es capaz de adoptar el punto de vista de un ladrón para conseguir descifrar su comportamiento sin dejar de ser por ello un policía. El policía es, actúa y piensa como tal, mantiene su punto de vista, y simplemente intenta poder pensar como un ladrón, entender su perspectiva, para poder capturarlo. Por tanto, podemos ver que para Moline, un punto de vista puede aparecer de dos maneras: uno puede tomar un punto de vista o bien puede

14 En lo que sigue, resumiré la tesis de Moline (1968, pp. 191-198). 
intentar comprender el punto de vista de otro, algo que puede ser observado en literatura, donde encontramos el propósito de caracterizar ciertos puntos de vista. Para él (Moline, 1968, 195) "adoptar un punto de vista" puede, por tanto, entenderse de diferentes formas desde la perspectiva filosófica: entender un punto de vista dado, caracterizar o evaluar un punto de vista de forma crítica o adoptarlo, es decir, tener un punto de vista propio desde el cual algo es enfocado.

Así, Moline analiza diferentes clases de afirmaciones y preguntas que pueden estar asociados a puntos de vista y que nos permiten identificarlos desde una perspectiva filosófica. Se trata de criterios muy abiertos que nos permiten identificar la forma peculiar en la que se ve el mundo desde un determinado punto de vista, a pesar de que no podamos derivar de éstos definiciones precisas ni condiciones necesarias o suficientes para encasillarlos. La adopción de un punto de vista en una afirmación comprensiva (comprehension claims) puede verse al intentar entender un punto de vista, por ejemplo, el del físico Robert Oppenheimer cuando creó la bomba atómica, lo cual requeriría no solo de la comprensión de las expectativas y oportunidades disponibles en el conocimiento de la época, sino que requeriría, por ejemplo, justificar y comprender su creación por las exigencias del Estado americano, esto es, por el contexto político y social. Por otro lado, las afirmaciones de que una determinada consideración es relevante o irrelevante desde algún punto de vista u otro constituyen una segunda clase. Moline nos dice que serían, por ejemplo, aquellas cuestiones psicológicas que pueden parecer irrelevantes desde un punto de vista filosófico. A este tipo podemos llamarlo algo así como afirmaciones de irrelevancia (irrelevance claims). Otras veces encontramos, según este autor, afirmaciones más bien críticas hechas también mediante de la expresión punto de vista como, por ejemplo, "El senador Yawn decidió tomar un punto de vista estrecho y económico sobre la legislación" (Moline, 1968, 193) en este caso, la afirmación sobre el punto de vista tiene que ver con la amplitud o estrechez con la cual somos capaces de enfocar un determinado tema. Esta forma de referirnos a un punto de vista, será denominada por Moline como afirmaciones de tamaño (size claims).

Hasta ahora hemos visto de qué forma nos referimos a los puntos de vista y que significado atribuimos a estos con nuestras afirmaciones. Con todo, el trabajo de Moline va aún un poco más allá, y es que este autor afirma que los puntos de vista tienen bastante que ver con la personalidad y el rol, en tanto que encontramos diferentes formas de enfoque sobre la realidad en función del grupo en que nos fijemos. Para mostrar esta idea, Moline piensa en el punto de vista que puede tener un físico, un administrativo, un padre o un gobierno, pero también a entidades inanimadas pueden serles atribuidas perspectivas, podemos, por ejemplo, referirnos al punto de vista del cine, del rock y el de una plaza o, incluso, aludir al punto de vista de un perro, de un ordenador o hasta el de un gusano. Podemos ponernos en o imaginarnos, todos estos puntos de vista. Moline cae en la cuenta de que, por lo general, nos parece mucho más fácil ponernos en el lugar de un perro que en el de, por ejemplo, una almeja. Esto podría ocurrir porque tenemos una mayor familiaridad con el perro y porque podemos asemejarlo a nosotros. En cierta forma podemos ver a nuestro perro como una persona en tanto que nos muestra una cierta personalidad, y esto parece imprescindible para poder referirnos o hacernos una idea de lo que es un punto de vista.

Otra forma de aproximarnos a lo que supone esta expresión implica fijarnos en que, a menudo, podemos encontrar una conexión entre tomar un punto de vista y tener una posi- 
ción en el sentido de desempeñar un papel. Todas las entidades, grupos o personas, están asociadas, según Moline, a ciertos roles. En función de la variedad de roles que adoptemos tendremos un punto de vista diferente y de cierta forma restrictivo; no es lo mismo ser un consumidor de tecnología que ser productor de ésta. Como vemos, el punto de vista, además de estar asociado a la personalidad, lo estará también al rol, el puesto que ocupemos. Para entender un punto de vista se hace necesario entender los diversos roles de alguien, aunque esto no nos asegure un completa comprensión de su punto de vista, podemos, por ejemplo, hablar del punto de vista de alguien que sufre una enfermedad mental, aunque no podamos comprender del todo qué implica estar en su papel. Por tanto, entiende que la conexión entre los roles y el punto de vista no es demasiado fuerte, pero sí que puede arrojar luz acerca de lo que es un punto de vista. Moline recurre al teatro como un lugar en el que podemos ver qué es un rol y cómo influye en un punto de vista, en tanto que en él se crea el rol, la personalidad, a la que da vida a un actor y que conforma lo que debe sentir, hacer o decir un personaje. Por lo general, cuando decimos que adoptamos un determinado rol nos estamos refiriendo a simular la posición de otra persona. Tener un punto de vista y ocupar un puesto parece implicar actuar o comportarse de cierta manera. Por lo tanto, la consideración sobre la conexión entre roles y puntos de vista le hace reemplazar su pregunta original “¿Qué es tomar un punto de vista?” por “¿Qué hace uno al tomar un punto de vista?”. La respuesta a esta pregunta estaría asociada al conjunto de aquellos rasgos y factores que se hacen relevantes a la hora de tomar decisiones desde un determinado punto de vista.

Moline concluye, de esta forma, que tomar un punto de vista es semejante a mirar a través de una lente construida para un propósito particular, con un foco y campo de visión particular. Algunos objetos estarán enfocados, se hacen relevantes, y otros simplemente serán excluidos puesto que no son importantes desde una determinada perspectiva. En general, Moline considera que adoptar un punto de vista conlleva ciertas tendencias, como perseguir ciertos intereses y objetivos específicos, utilizar ciertos criterios para la evaluación de acciones que conducen o no al logro de estos objetivos y considerar otros intereses o criterios como irrelevantes. Hacer suposiciones fácticas acerca de lo que supone ser, por ejemplo, un científico, tendría que ver, en este caso, con las creencias y el método que caracterizan su trabajo y su forma de enfocar el mundo. Así, tomar un punto de vista supone una tendencia a compartir intereses, metas, criterios, suposiciones y juicios de relevancia o irrelevancia característicos de quienes toman dicho punto de vista. Decir que más de una persona adopta un punto de vista implica que se comparten estas características o al menos actuar como si esto ocurriese.

Moline dice que la expresión "punto de vista" no parece usarse simplemente para describir ciertos eventos mentales, reales o ideales, sino que es utilizada en la reconstrucción racional, la interpretación y la crítica de las tendencias racionales e irracionales en el comportamiento. Los criterios relevantes y las suposiciones fácticas tendrían también relevancia al hablar de un punto de vista moral. En un punto de vista existen objetivos, criterios, suposiciones y cursos de acción que pueden ser defendidos o prohibidos. Así, nos dice Moline, en ocasiones, puede ocurrir que un determinado punto de vista moral pueda coincidir con el punto de vista del interés propio. Para demostrar esto se debe revisar, a la luz de los hechos de un caso concreto, los intereses relevantes, los criterios, suposiciones y cursos de acción para mostrar qué aspectos se comparten; y estos mismos factores deberían ser revisados 
si pensamos que dos puntos de vista son incompatibles. Lo mismo ocurriría con las afirmaciones de comprensión, de irrelevancia o de tamaño que vimos anteriormente. Cuando intentamos ponernos en el punto de vista de un científico, por ejemplo, Oppenheimer, vemos cómo tenemos en cuenta estos factores en nuestro intento de comprender sus actos y comportamientos. Recurriremos a suposiciones, intereses, objetivos, criterios y restricciones con respecto a lo que era o no relevante. Como vemos, estos factores parecen imprescindibles a la hora de explicar el comportamiento humano y, según Moline, conocerlos puede incluso permitirnos predecir el comportamiento futuro.

En definitiva, Moline entiende que adoptar un de punto de vista puede tener diferentes significados: entender un punto de vista dado, caracterizar o evaluar un punto de vista de forma crítica o adoptarlo, es decir, tener un punto de vista propio desde el cual algo es enfocado. Según este autor, adoptar un punto de vista tiene que ver, más que con principios, con una determinada vivencia, por lo que podemos entender que, en las series de televisión, como en la literatura o el cine, se nos permite adoptar experiencias o vivencias que nos resultan lejanas. Se nos muestra, por ejemplo, el punto de vista o rol de un ingeniero y un programador informático, como en el caso de Halt and Catch Fire ${ }^{15}$, el punto de vista de un cirujano a principios del siglo XX como ocurre en The Knick o las diferentes perspectivas a la hora de abordar una controversia tecnológica, como veremos a continuación a través de un capítulo de Black Mirror.

\section{Puntos de vista científicos en el caso de Black Mirror}

A continuación, intentaré ejemplificar esta forma de democratización del conocimiento y la proyección de los puntos de vista en una serie de televisión utilizando un ejemplo de la ya mencionada Black Mirror. A través del capítulo White Bear ${ }^{16}$ veremos cómo a través de diferentes puntos de vista el espectador se forma una imagen sobre el desarrollo de una determinada tecnología. Para explicarlo, deberemos atender primero a su trama. El capítulo comienza con un timbre molesto cuyo origen desconocemos y con una masa de gente que parece no poder evitar el uso de su Smartphone para grabar a una mujer que está en peligro, Victoria. En este momento, observamos una perspectiva, la de la víctima, que huye ante la pasiva mirada de la multitud que se limita a grabarla. Luego, la trama cambia y el espectador amplía su perspectiva al entender que la víctima es parte de un montaje televisivo que pretende hacer justicia a un crimen que ésta ha cometido. El público comienza a comprender entonces que la protagonista, por la que ha sufrido empáticamente durante el fatigoso capítulo, había encubierto el asesinato de una niña a la que su marido secuestró y asesinó. Así, la mujer a la que considerábamos víctima comienza a ser vista como verdugo en el momento en que nos enteramos de que habría encubierto un asesinato y que, además, lo había grabado.

Lo que vemos con esta estructura narrativa son diferentes puntos de vista. En Liz (2012) se nos muestra una forma de distinguir entre diferentes puntos de vista y, para explicar la forma del relato de este capítulo, propongo recurrir a algunos de ellos fijándonos en el titular, esto es, en los diferentes portadores de los puntos de vista que constituyen la narración y que,

15 AMC: 2014-2017.

16 Episodio dos de la tercera temporada. Dirigido por Carl Tibbetts y estrenado el 18 de febrero de 2013. 
a su vez, nos permitirá observar el conflicto ético en esta controversia. En general podemos acordar que, aunque no provenga de un sujeto, esta serie muestra un punto de vista; supone una visión un tanto pesimista sobre la tecnología y nuestra relación con ella. A través de un futuro distópico se nos muestra un posible desarrollo de la ciencia y la tecnología que nos pervierte. A su vez, en este capítulo concreto encontramos dos puntos de vista diferentes que provienen de diferentes sujetos o entidades. En primer lugar, tenemos el punto de vista individual de la protagonista, esto es, un punto de vista personal que nos muestra su subjetividad y su modo de entender la situación que vive. Victoria no recuerda quién es ni por qué despierta en un lugar donde todo parece estar controlado por un monitor, desde su perspectiva el resto son los "malos", representan un peligro. Esto constituye su punto de vista personal, y durante la primera parte del capítulo, el espectador, de forma intersubjetiva, lo comparte, sólo conoce el punto de vista del cual Victoria es titular. Es decir, nuestro punto de vista se involucra con el de Victoria. Sólo en la última parte del capítulo el espectador comienza a ser partícipe del punto de vista colectivo que muestra la sociedad en la que despierta la protagonista y que entiende que la enemiga es Victoria. Se trataría de un punto de vista colectivo en tanto que se nos presenta el punto de vista de una colectividad de sujetos, más allá de si podemos encontrar o no en ésta una vida mental independiente. Cuando el espectador comienza a tener un punto de vista intersubjetivo al respecto de la colectividad, comienza a encontrar respuestas a todas aquellas preguntas que se había formulado mientras seguía la perspectiva de Victoria. Las paredes del transmisor se abren y muestran al público sentado en un plató de televisión, sólo entonces somos capaces de entender qué ha ocurrido de una forma objetiva. Previamente nuestra perspectiva era cercana al carácter experiencial de un sujeto, es decir, nuestro punto de vista es relativo a cómo la protagonista está situada de una determinada forma en el mundo. Así, a través de este capítulo la serie nos propone pensar la problemática ética que surge del desarrollo y el uso de la tecnología desde dos puntos de vista. El primero es el personal y subjetivo, que muestra la experiencia de Victoria, el segundo es el de la colectividad, la sociedad a la que identificamos a partir de su intención de utilizar la tecnología para ejecutar una venganza macabra. En la primera parte, los malvados son la sociedad y la gente que persigue a la protagonista mientras que, en la segunda, a partir de la nueva información, pasamos de ver a Victoria como víctima para entender que es una delincuente. El efecto que produce sobre el espectador este capítulo generaría, a su vez, un tercer punto de vista capaz de unificar ambas y que reflexiona sobre el problema ético de esta terrible utilización de la tecnología.

Además de la proyección de diferentes puntos de vista de los personajes, en una serie de televisión, encontramos, en primer lugar, un punto de vista principal: la cámara. El medio técnico que permite grabar una serie de televisión es en sí mismo una focalización de las escenas. Como nos decía Moline, un punto de vista puede ser encontrado en la cámara. A través de ella se consigue que el espectador vea imágenes que han sido seleccionadas específicamente para generar una imagen concreta desde un determinado emplazamiento. Así, las series adquieren una función comprensiva mediante la retransmisión de diferentes puntos de vista que son o pueden ser tomados con respecto a una determinada controversia. Digamos que, al igual que existen visiones que sólo puede tener una persona que se encuentre en lo más alto de una montaña, existen cuestiones relacionadas con la ciencia y la tecnología que no son consideradas más que por científicos o, en todo caso, por comités éticos, en cual- 
quier caso, podríamos decir que pertenecen también a lo "más alto" del mundo académico o investigador. En una serie de televisión encontramos afirmaciones comprensivas, pero no sólo eso, sino que también se presentan afirmaciones de irrelevancia o incluso de tamaño. Esto puede ser también observado en el episodio White Bear, donde podemos observar cómo desde cada perspectiva, la de Victoria o en la de la sociedad, se toman por relevantes cosas diferentes. Para Victoria lo relevante será su vida, salvarse, para la sociedad lo principal es conseguir hacer del crimen un espectáculo. Para ambos el enfoque de la situación es distinto, y también lo será la amplitud con la que observan los hechos, digamos que el punto de vista de la protagonista puede ser entendido como más estrecho en tanto que no es consciente de la dinámica a la que obedece la situación que está viviendo ni tampoco recuerda el crimen por el que está pagando, mientras que el resto sí y, precisamente por ello, nadie la ayuda. Como vemos, en este capítulo se permite observar cómo influye la personalidad, el rol, en el punto de vista desde el cual se enfoca una determinada situación. Las suposiciones, intereses, objetivos, criterios y restricciones que aparecen en cada uno de los puntos de vista son diferentes, y al contraponerlas se nos muestra este peculiar dilema o controversia con respecto al uso de la tecnología.

\section{Conclusiones}

Hemos podido comprobar como una serie de televisión como Black mirror muestra, a través de diferentes puntos de vista, una controversia con respecto a la utilización y desarrollo de la tecnología. El espectador en este capítulo es capaz de recibir información relevante de los dos bandos: Victoria y la sociedad, y reflexionar a partir de ahí sobre qué uso debe tener la tecnología o si es legítima la utilización de esta en cualquier situación. En el relato que encontramos en una serie de televisión se proyectan, por lo general, diferentes puntos de vista que nos permiten revelar en qué consiste una controversia científica. Si, como decía Moline, analizar y comprender puntos de vista permite predecir, podríamos estar ante herramienta muy ilustrativa sobre la práctica científica o los factores que determinan la dirección de las investigaciones. En esta ocasión, Black mirror hiperboliza la utilización de las tecnologías con las que contamos en la actualidad para mostrarnos cómo un uso desorbitado de, por ejemplo, las redes sociales pueden tener consecuencias aterradoras. En este capítulo hemos podido observar la utilización del perspectivismo para narrar un relato que versa sobre las diferentes formas de enfocar las consecuencias de ciertos usos de la tecnología y las diferentes vivencias a las que da lugar.

En definitiva, las series de televisión pueden ser entendidas como una forma de acercar problemas a los espectadores y, en este sentido, muestran un carácter democratizador. Podríamos, incluso, decir que las series han adquirido la potencialidad emancipadora de la que nos hablaba Adorno; son capaces de plasmar la realidad y cuestionarla. A través de los diferentes puntos de vista que son proyectados en una serie de televisión se permite que el público comprenda determinadas controversias e incorpore las necesidades de los otros en la reflexión acerca del desarrollo científico. Las nuevas tecnologías aparecen en nuestras vidas sin haber tenido demasiado tiempo para reflexionar sobre ellas. Ante este panorama, las series proporcionan una forma de proyectar los diferentes puntos de vista relacionados con el uso de la ciencia y la tecnología. El público es instruido sobre ciertas prácticas, dilemas y 
conceptos relacionados con la ciencia; facilitan el conocimiento necesario para convertirlos en deliberadores. Podemos concluir diciendo que las series de televisión, siendo un producto cultural, pueden convertirse en una alternativa que permita formar una opinión responsable sobre conocimiento científico o, al menos reflexionar sobre las posibles aplicaciones tecnológicas. Encontramos, por tanto, un carácter filosófico en las series de televisión; éstas nos permiten, como decíamos, reflexionar sobre el mundo y nuestra relación con él o, incluso, sobre la ciencia y la forma en que su desarrollo nos repercute.

\section{Referencias}

Adorno, Th.W. y H. Eisler (1947), Composing for the Films, New York: Oxford University Press. Citas de la edición castellana, El cine y la música, Madrid: Ed. Fundamentos, 2015.

Barceló García, M. (2005), “Ciencia y ciencia ficción”, Revista Digital Universitaria, vol. $6, \mathrm{n}^{\circ} 7$.

Bourdieu, P. (1996), Sur la télévision: duivi de L'emprise du journalism, Paris: Liber éditions. Citas de la edición castellana Sobre la televisión, Barcelona: Anagrama, 2016.

Cascajosa, C. (2016), La cultura de las series, Barcelona: Laertes.

Colomina-Almiñana J.J. (2018), Formal Approach to the Metaphysics of Perspectives, Synthese Library (Studies in Epistemology, Logic, Methodology, and Philosophy of Science), Heidelberg: Springer.

Kitcher, Ph. (2010), "Science in a Democratic Society”, en: González, W.J. (ed): Scientific Realism and Democratic Societies: The Philosophy of Philip Kitcher, Poznan Studies in the Philosophy of Science and the Humanities, Amsterdam: Rodopi, pp. 95- 112.

Liz, M. (2012), Puntos de vista. Una investigación filosófica, Barcelona: Laertes.

Martin, B. (2014), Hombres fuera de serie, Barcelona: Ariel.

Moline, J. (1968), “On points of view”, American Philosophical Quaterly, University of Illinois Press on behalf of the North American Philosophical Publications, vol. 5, pp. 191-198.

Ortega y Gasset, J. (1923), El tema de nuestro tiempo, Madrid: Calpe. Citas de la edición de Madrid: El arquero, 1970.

Rancière, J. (2008), Le spectateur émancipé, Paris: La fabrique éditions. Citas de la edición castellana, El espectador emancipado, Ellago Ensayo, 2010.

Vázquez, M. y M. Liz (2015), Temporal Poins of view. Subjective and objective aspects. Heildelberg: Springer

Wolton, D. (1999), Internet et après. Une théorie critique des nouveaux médias, Paris: Flammarion. Citas de la edición castellana, Internet, ¿y después? Una teoría crítica de los nuevos medios de comunicación, Barcelona: Gedisa, 2000. 